Post-print of: Bauer, Thomas et al. "The Halphen cubics of order two" in Collectanea mathematica (2016). The final version is available at DOI 10.1007/s13348-0160172-4.

\title{
The Halphen cubics of order two
}

\author{
Thomas Bauer, Brian Harbourne, Joaquim Roé, Tomasz Szemberg
}

May 13, 2016

\begin{abstract}
For each $m>1$, Roulleau and Urzúa give an implicit construction of a configuration of $4\left(3 \mathrm{~m}^{2}-1\right)$ complex plane cubic curves. This construction was crucial for their work on surfaces of general type. We make this construction explicit by proving that the Roulleau-Urzúa configuration consists precisely of the Halphen cubics of order $\mathrm{m}$, and we determine specific equations of the cubics for $m=1$ (which were known) and for $\mathrm{m}=2$ (which are new).
\end{abstract}

\section{Introduction}

For each $n=3 m$, we study certain arrangements of $\frac{4}{3}\left(n^{2}-3\right)$ plane cubic curves; each curve is isomorphic to the Fermat cubic $x^{3}+y^{3}+z^{3}$ (i.e., to $T=C / Z[\square], \square=e^{2 \pi i / 6}$ ). For $\mathrm{n}=3$, the eight curves in the arrangement were known from invariant theory [2] and give the "inscribed and circumscribed" cubics [1, Proposition 5.2] for the four singular cubics in the Hesse pencil $h x^{3}+y^{3}+z^{3}, x y z i$, but for $m>1$ the arrangements are described explicitly here for the first time.

These arrangements come from arrangements of $4 n^{2}$ elliptic curves on the abelian surface $T \times T$, studied by Hirzebruch in [13]. Using a quotient by a finite group action followed by a blow up to resolve singularities, Roulleau and Urzúa obtain in [16, section 3] corresponding arrangements of $\frac{4}{3}\left(n^{2}-3\right)$ elliptic curves on a rational surface they call $\mathrm{H}$, whose images (see [15, Section 1]) under a birational morphism to $\mathrm{P}^{2}$ give the arrangements of plane cubics we study here. The Roulleau-Urzúa arrangements on $\mathrm{H}$ were crucial for their construction of surfaces of general type in [16]. In [15], Roulleau shows that the corresponding arrangements of plane cubics are interesting for another reason: the Harbourne index of the union $C_{n}$ of the

\footnotetext{
Acknowledgements: The understanding necessary for this paper grew out of discussions that occurred in working groups at two workshops in 2015, namely "Recent advances in Linear series and Newton-Okounkov bodies", February 9-14 at the University of Padua, and "Ideals of Linear Subspaces, Their Symbolic Powers and Waring Problems", February 15-21 at the Mathematisches Forschungsinstitut Oberwolfach. We thank the participants in these workshops and the institutions that hosted them. We also thank the University of Freiburg for hosting visits by Bauer and Harbourne to work on this paper with Szemberg in person for a week in the summer of 2015. In addition, the research of Bauer was partially supported by DFG grant BA 1559/6-1, the research of Roé was partially supported by MTM 2013-40680-P (Spanish MICINN grant) and 2014 SGR 114 (Catalan AGAUR grant), and the research of Szemberg was partially supported by National Science Centre, Poland, grant 2014/15/B/ST1/02197

Keywords: Automorphisms, abelian surfaces, Halphen cubics, Hesse arrangement, rational surfaces Mathematics Subject Classification (2010): 14C20, 14E05, 14H52, 14J26, 14K12.
} 
$\frac{4}{3}\left(n^{2}-3\right)$ cubics has limit -4 as $n \rightarrow \infty$. We note that no reduced plane curve is yet known with Harbourne index less than or even equal to -4 .

The arrangements of cubics as given in [15] are not given explicitly; they are described only as images under birational maps. In this paper we construct the same arrangements of cubics by elementary methods directly on $\mathrm{P}^{2}$, which allows us to give explicit equations for these interesting cubics. In the case $n=3$, the corresponding eight cubics coincide with the classical Halphen cubics $[11,1,2,10]$. For $n=3 m$ with $m>1$, they are members of four pencils, where each pencil is spanned by two of the eight Halphen cubics. We call these pencils Hesse singular point cubic pencils. (The reason for this name is that these pencils can also be obtained from the singular points of the four singular cubics in the Hesse pencil. Each of these singular cubics has three singular points. Thus each choice of three of the four singular cubics in the Hesse pencil defines 9 points, and these 9 points are the base points of a cubic pencil. The four pencils defined this way are precisely the four pencils obtained from the eight Halphen cubics.) The specific members chosen from each Hesse singular point cubic pencil depend on the $3 \mathrm{~m}$-torsion points of $\mathrm{T}$, so we refer to the specific cubics chosen as the Halphen cubics of order $\mathrm{m}$.

Our construction relies on classical facts known for the so-called dual Hesse configuration of 9 lines in the plane meeting by threes on 12 points, and on the geometry of the curve $\mathrm{T}$. The Weierstrass function $\wp^{0}$ is a morphism to $\mathrm{P}^{1}$ of degree 3 , which in the case of $T$ is triply ramified at 3 points. For each positive integer $\mathrm{m}$, we denote by $\mathrm{P}^{1}[\mathrm{n}]$ the images of the $\mathrm{n}$-torsion points of $\mathrm{T}$. As noted above, the Hesse singular point cubic pencils are defined by taking as base points subsets of 9 points among the 12 vertices of the dual Hesse configuration; each pencil has 3 reducible members which are composed of lines of the configuration. We parameterize the pencils so that the reducible members correspond to parameters $\mathrm{u} \in \mathrm{P}^{1}$ belonging to the branch locus of $\wp^{0}$. Then the Halphen cubics of order $m$ are defined as the cubics in the Hesse singular point cubic pencils with parameters in $\mathrm{P}^{1}[3 \mathrm{~m}]$. Our first result is the following:

Theorem 1 For each $n \in 3 N$, let $H(n)$ be the union of all Halphen cubics of order $n / 3$. The singularities of $H(n)$ are: 12 points of multiplicity $n^{2}-3$ at the vertices of the dual Hesse configuration, with $n^{2} / 3-1$ triple points infinitely near to them, and $\left(n^{2}-3\right)\left(n^{2} / 3-3\right)$ quadruple points.

As a corollary the Harbourne index of $H(n)$ tends to -4 as $n$ grows. The configuration of Halphen cubics therefore behaves like the Roulleau-Urzúa configuration over which it is modeled. Our second goal is to understand the rational map $\mathrm{T} \times \mathrm{T} 99 \mathrm{~K} \mathrm{P}^{2}$ used by Roulleau and Urzúa to construct their configuration. In Theorem 2.1 we determine the linear series associated to the map, and using the action of the theta group, we prove that both configurations agree:

Theorem 2 The curves that form the Roulleau-Urzúa configuration corresponding to the $n$-torsion points for $n=3 m$ are the Halphen cubics of $m$-th order.

It is worth noting that the degree 3 rational map $\mathrm{T} \times \mathrm{T} 99 \mathrm{~K} \mathrm{P}^{2}$ had been studied classically by Comessatti [8]; see [6] for a modern account.

The paper is organized as follows. In section 1 we recall the classical construction of Hesse line configurations and Halphen cubics, and we prove Theorem 1 along with additional information on the position of the singularities (Theorem 1.5). Section 2 is 
devoted to the study of the Roulleau-Urzúa configuration and the map $\mathrm{T} \times \mathrm{T} 99 \mathrm{~K} \mathrm{P}^{2}$, and culminates in the proof of Theorem 2.

\section{The Hesse configurations and Halphen cubics}

Recall the construction of the so-called Hesse configurations (a modern account of this classical subject can be found in the book [9, Section 3.1], see also [1] and references therein for their history and attributions).

Given a smooth plane cubic $C$ and a line 'joining two flexes of $C$, the third intersection of ' and $C$ is another flex. Altogether, there are 12 such lines of flexes, and at each of the 9 flexes of $C$ exactly 4 of the 12 lines meet. This configuration of lines and points is classically called the Hesse line arrangement $\left(12{ }_{3}, 9_{4}\right)$; it does not depend on the choice of a cubic, i.e., the sets of 9 flex points of any smooth plane cubic are projectively equivalent.

A triangle containing all 9 flexes is called a triangle of flexes; there are 4 such triangles which together form the Hesse arrangement. They can be obtained as follows. Fix one of the flexes $p_{0}$ as the zero for the group law on $C$; then the 9 flexes of $C$ form the 3-torsion subgroup $C[3] \cong(Z / 3 Z)^{2}$. Each line of flexes 'through $p_{0}$ cuts on $C$ one of its 4 cyclic subgroups of order 3 . The two cosets of this subgroup in $C[3]$ correspond to the two lines of flexes which do not meet ' on $C$, which together with ' form one of the triangles.

The polar curve of $C$ with respect to a flex $p$ is the degenerate conic consisting of the tangent $T_{p} C$ and another line, called the harmonic polar of $p$. If ' is a line of flexes, the harmonic polars of the three flexes on 'are concurrent, and their point of intersection is the vertex opposite to 'in the triangle of flexes to which it belongs. Thus, each of the 9 harmonic polars goes through 4 vertices, one on each triangle of flexes, and at each of the 12 vertices exactly 3 of the 9 lines meet. They form the dual Hesse arrangement $\left(9_{4}, 12_{3}\right)$, which again does not depend on the cubic.

The given curve $C$ and each triangle of flexes are cubics through the 9 flex points. It follows that the 9 points of the Hesse configuration are the base points of a pencil of cubics, called the Hesse pencil ; all cubics in the pencil have the same flex points, and every plane cubic is projectively equivalent to one of the curves in the pencil. The Hesse pencil has 4 singular members, namely the 4 triangles of flexes. We denote them $T_{v}, T_{h}, T_{\delta}, T_{\gamma}$. For convenience we also fix the following notations for the whole paper: $T=\{v, h, \square, \square\}$ will be the set of indices for the triangles; $V_{v}=\left\{v_{0}, v_{1}, v_{\infty}\right\}$ will be the vertices of $T_{v}$; and similarly for $V_{h}=\left\{h_{0}, h_{1}, h_{\infty}\right\}, V_{\delta}=\left\{\square_{0}, \square_{1}, \square_{\infty}\right\}$ and $V_{\gamma}=\left\{\square_{0}, \square_{1}, \square_{\infty}\right\}$ (the symbols $V, h, \square, \square$, and $0,1, \infty$, are chosen to match

with whole set of vertices of the dual Hesse configuration, and for each $t \in T, \Lambda_{t}=V \backslash V_{t}$ to be the complement in $\mathrm{V}$ of the set of vertices of $T_{t}$.

The group of projective transformations of the plane which preserve the Hesse arrangement (or equivalently the Hesse pencil) is a finite group $G_{216}$ called the Hesse group. It obviously acts on the set $\left\{T_{v}, T_{h}, T_{\delta}, T_{\gamma}\right\}$, and the image of the corresponding representation $G_{216} \rightarrow S_{4}$ is the alternating group $A_{4}$. Since it is not the full $S_{4}$ group, the order of the indices $v, h, \square, \square$ is not entirely innocuous; we now introduce coordinates in order to be precise, and for later use in the explicit determination of the Halphen cubics.

Take $C$ to be the Fermat cubic $x^{3}+y^{3}+z^{3}$, and let $\square$ denote a primitive third root 
of unity. The 9 flex points of $C$ are the points $(-1,1,0),(-1, \square, 0)$ and $\left(-1, \square^{2}\right.$, 0 ) and the 6 other points obtained from these by permutation; it is customary to take as generators for the Hesse pencil the Fermat cubic $C$ and the triangle $T_{\mathrm{v}}=$ xyz. Thus the three coordinate points

$$
\mathrm{v}_{0}=(1: 0: 0), \quad \mathrm{v}_{1}=(0: 1: 0), \quad \mathrm{v}_{\infty}=(0: 0: 1)
$$

are three of the vertices of the dual Hesse configuration; the remaining points are

$$
\begin{aligned}
& \mathrm{h}_{0}=(1: 1: 1), \quad \mathrm{h}_{1}=\left(1: \square: \square^{2}\right), \quad \mathrm{h}_{\mathrm{u}}=\left(1: \square^{2}: \square\right) \\
& \mathrm{\square}_{0}=(\square: 1: 1), \quad \mathrm{Q}_{1}=(1: \square: 1), \quad \square_{\infty}=(1: 1: \square) \\
& \square_{0}=\left(\square^{2}: 1: 1\right), \quad \square_{1}=\left(1: \square^{2}: 1\right), \quad \square=\left(1: 1: \square^{2}\right)
\end{aligned}
$$

The equations of the harmonic polar lines forming the dual Hesse configuration can be obtained using the coordinates of the points. For example the line through the points $v_{0}$ and $h_{0}$ has equation $z-y$ and also goes through $\square_{0}$ and $\square_{0}$. We denote by $L_{i, j, k, 1}$ the line through $v_{u_{i}}, h_{u_{j}}, a_{u_{k}}$ and $\square_{u_{1}}$, so the line just considered is $L_{0000}$. Evaluating all collinearities we obtain

$$
\begin{array}{lll}
\mathrm{L}_{0000}=\mathrm{z}-\mathrm{y}, & \mathrm{L}_{01 \infty 1}=\mathrm{z}-\mathrm{Dy}, & \mathrm{L}_{0 \infty 1 \infty}=\mathrm{Dz}-\mathrm{y} \\
\mathrm{L}_{1011}=\mathrm{z}-\mathrm{x}, & \mathrm{L}_{110 \infty}=\mathrm{Dz}-\mathrm{x}, & \mathrm{L}_{1 \infty \infty 1}=\mathrm{z}- \\
\square \mathrm{x} & & \\
\mathrm{L}_{\infty 0 \infty \infty}=\mathrm{y}-\mathrm{x}, & \mathrm{L}_{\infty 110}=\mathrm{y}-\square \mathrm{x}, & \mathrm{L}_{\infty \infty 01}=\mathrm{y}- \\
\mathrm{x} & &
\end{array}
$$

For each $t \in T$, the linear system of cubics through the 9 points in $\Lambda_{t}$ is a pencil $C_{t}$, which we call a Hesse singular point cubic pencil. It has three singular members, namely, for each vertex $t_{u}$ of $T_{t}, u \in\{0,1, \infty\}$, the union of the three harmonic polars concurrent at $t_{u}$ is a member of the pencil, which we call $C_{t_{u}}$. All nonsingular members of each pencil have $\boldsymbol{j}$-invariant equal to zero. Halphen showed that the locus of 9-torsion points of all curves in the Hesse pencil consists of two members of these pencils $C_{h}, C_{v}, C_{\delta}, C_{\gamma}$ (so-called Halphen cubics); the configurations of plane cubics described by Roulleau and Urzúa consist of members of the same pencils (which we call higher order Halphen cubics).

Later on we shall give explicit equations of the Halphen cubics; for this purpose we fix a coordinate $u$ in each pencil $C_{t}$, such that $\{0,1, \infty\}$ correspond to the singular members. Since the harmonic polars concurrent at $t_{u}$, in the notation above, are the $L_{u_{v}} u_{h} u_{\delta} \quad u_{\gamma}$ with $u_{t}=u$, the three singular members of $C_{v}$ are

$$
\begin{aligned}
& C_{v_{0}}=u^{2} L_{0 * * *}=\square^{2}(z-y)(z-\square y)(\square z-y)=z-3 y \\
& C_{v_{1}}={ }^{2}{ }_{L_{1 * * *}}=\square^{2}(z-x)(\square z-x)(z-\square x)=\xi-z \\
& C_{V_{\infty}}=a^{2}{ }_{L_{\infty * * *}}=\square^{2}(y-x)(y-\square x)(\square y-x)=y^{3}-x^{3}
\end{aligned}
$$

where the factor $\square^{2}$ serves only a simplification purpose. Similarly, the three singular members of $C_{h}$ are

$$
\begin{aligned}
& \mathrm{C}_{\mathrm{h}_{0}}=\quad \mathrm{L}_{* 0 * *}=(\mathrm{z}-\mathrm{y})(\mathrm{z}-\mathrm{x})(\mathrm{y}-\mathrm{x})= \\
& -x^{2} y+x y^{2}+x^{2} z-y^{2} z-x z^{2}+y z^{2} \\
& C_{\mathrm{h}_{1}}=-\mathrm{L}_{\star 1 * *}=-(\mathrm{z}-\square \mathrm{y})(\square z-\mathrm{x})(\mathrm{y}-\square \mathrm{x})= \\
& u^{2}\left(x^{2} y-a^{2} x y^{2}-a^{2} x^{2} z+y^{2} z+x z^{2}-a^{2} y z^{2}\right) \\
& C_{\mathrm{h}_{\infty}}=\quad \mathrm{Y}_{\mathrm{L}_{\star \omega \times x}}=(\square z-\mathrm{y})(\mathrm{z}-\square \mathrm{x})(\square \mathrm{y}-\mathrm{x})= \\
& -\square\left(x^{2} y-\square x y^{2}-\square x^{2} z+y^{2} z+x z^{2}-\square y z^{2}\right)
\end{aligned}
$$


where the signs are chosen so that $C_{h_{0}}+C_{h_{\infty}}=C_{h_{1}}$. Finally, the singular members of $\mathrm{C}_{\delta}$ and $\mathrm{C}_{\gamma}$ are

$$
\begin{aligned}
& C_{\delta_{0}}=\quad{ }_{L_{* \star 0 *}}=(z-y)(\square z-x)(\square y-x)= \\
& -x^{2} y+\square x y^{2}+x^{2} z-\square^{2} y^{2} z-\square x z^{2}+\square^{2} y z^{2} \\
& C_{\delta_{1}}=-{ }^{Y} L_{* * 1 *}=-(z-\square y)(z-\square x)(y-x)= \\
& \square^{2}\left(x^{2} y-x y^{2}-\square^{2} x^{2} z+\square^{2} y^{2} z+\square x z^{2}-\square y z^{2}\right) \\
& C_{\delta_{\infty}}=\quad \mathrm{Y}_{\mathrm{L}_{\star \star \mathrm{w} \times \mathrm{x}}}=(\mathrm{Dz}-\mathrm{y})(\mathrm{z}-\mathrm{x})(\mathrm{y}-\mathrm{Dx})= \\
& -\square\left(x^{2} y-\square^{2} x y^{2}-\square x^{2} z+\square^{2} y^{2} z+\square x z^{2}-y z^{2}\right) \\
& C_{\gamma, 0}=P_{L_{* * * 0}}=(\square z-y)(\square z-x)(y-x)= \\
& -x^{2} y+x y^{2}+\square x^{2} z-\square y^{2} z-\square^{2} x z^{2}+\square^{2} y z^{2} \\
& C_{\gamma, 1}=-{ }^{Y} L_{* * * 1}=-(z-\square x)(y-\square x)(z-y)= \\
& \square^{2}\left(x^{2} y-\square^{2} x y^{2}-x^{2} z+\square y^{2} z+\square^{2} x z^{2}-\square y z^{2}\right) \\
& C_{\gamma, \infty}=\quad{ }_{L_{* * * \infty}}=(z-\square y)(z-x)(\square y-x)= \\
& -\square\left(x^{2} y-\square x y^{2}-\square^{2} x^{2} z+\square y^{2} z+\square^{2} x z^{2}-y z^{2}\right)
\end{aligned}
$$

With these choices, the member of the pencil $C_{t}$ corresponding to the parameter $\mathrm{u} \in \mathrm{C}$ will be $\mathrm{C}_{\mathrm{t}_{\mathrm{u}}}=\mathrm{C}_{\mathrm{t}_{0}}+\mathrm{u} \mathrm{C}_{\mathrm{t}_{\infty}}$ for every $\mathrm{t} \in \mathrm{T}$.

Halphen's Theorem. Fixing any of the flex points of a plane cubic curve $C$ as the zero point in the group law, the set of $n$-torsion points for $n=3 m$ coincides with the set of points $p$ such that there exists a (possibly reducible) curve of degree $\mathrm{m}$ meeting $\mathrm{C}$ only at $\mathrm{p}$; thus the set of $3 \mathrm{~m}$-torsion points of a cubic is well defined and independent of the choice of a flex. Halphen [11] studied the locus of points of $3 m$-torsion of all cubics in the Hesse pencil. For $m=1$ it consists on the 9 base points, as already said. For $\mathrm{m}=2$, it is the union of the 9 harmonic polars. For $\mathrm{m}=3$ it is the union of 8 cubics, two in each pencil $C_{u}$, which we shall describe next. For $m>3$, it is the union of 8 or 9 curves of higher degrees, depending on the divisibility of $m$ by 3 .

Lemma 1.1 Let $\mathrm{T}$ be an elliptic curve and $\mathrm{f}: \mathrm{T} \rightarrow \mathrm{P}^{1}$ a morphism of degree 3 with triple ramification at 3 points. Then the $\mathbf{j}$-invariant of $T$ is 0 , and $\mathbf{f}$ is unique up to translation and inversion on $\mathrm{T}$, and up to automorphisms of $\mathrm{P}^{1}$.

Proof. By the Riemann-Hurwitz formula, there is no more ramification than the three triple points, so $\mathrm{E}$ is a 3 sheeted cover of $\mathrm{P}^{1}$ away from these points. Permuting the sheets of the cover induces an automorphism of $E$ of order 3. But the only elliptic curves with an order 3 automorphism are those of $\mathbf{j}$-invariant 0 , and these support a single order 3 automorphism (up to translation and inversion), namely complex multiplication by a cube root of 1 [17, Theorem 10.1].

The automorphisms of $\mathrm{P}^{1}$ which appear in lemma 1.1 must obviously preserve the branch locus of $\mathbf{f}$. To take care of these we call marked line a pair $\mathrm{P}_{M}^{1}=\left(\mathrm{P}^{1}, \mathrm{M}\right)$ where $M=\{p, q, r\}$ is a set of 3 distinct points in $P^{1}$. A morphism of marked lines is an algebraic morphism which preserves the markings (and so any two marked 
lines are isomorphic). The group of automorphisms of the marked line is finite, isomorphic to $\mathrm{S}_{3}$, and by choosing a coordinate $\mathrm{u}$ on $\mathrm{P}^{1}$ such that the marking is $M=\{0,1, \infty\}$, it is generated by the morphisms $u 7 \rightarrow u^{-1}$ and $u 7 \rightarrow 1-u$. Then,

given an equianharmonic elliptic curve $\mathrm{T}$ and a marked line $\mathrm{P}_{\mathrm{M}}^{1}$, there exists a morphism $f: T \rightarrow \mathrm{P}^{1}$ of degree 3 , with triple ramification over $\mathrm{M}$, unique up to translation and inversion on $T$, and up to the action of $S_{3}$ on $\mathrm{P}_{\mathrm{M}}^{1}$.

In classical terminology, elliptic curves with $\mathbf{j}$-invariant equal to zero are called equianharmonic. In the setting of lemma 1.1, consider the group law on $T$ with the zero at one of the ramification points. Such a curve $T$ is obtained as $T=$ $\mathrm{C} / \mathrm{Z}[\mathrm{Q}]$, where $\mathrm{Q}=\mathrm{e}^{2 \pi \mathrm{i} / 6}$; a degree 3 map is given by the Weierstrass derivative function $\wp^{0}: \mathrm{T} \rightarrow \mathrm{P}^{1}$; and the corresponding order three automorphism is induced by multiplication by $\square=\square^{2}$. Its 3 fixed points, which are the ramification points

of $\wp^{0}$, are $p_{k}=\frac{k}{3}+\frac{k}{3} \square$ for $06 k 62$. Their images form the branch locus $M=$ $\operatorname{branch}(\mathbf{f})=\{i,-i, \infty\} \subset P^{1}$.

For every choice of the zero at one of the ramification points of $\mathbf{f}$, the other two ramification points are of 3-torsion (in fact they form a cyclic subgroup of order 3, as can be seen in the description via the $\wp^{0}$ function) hence for every positive integer $\mathrm{m}$ the set $\mathrm{T}[3 \mathrm{~m}]$ of $3 \mathrm{~m}$-torsion points on $\mathrm{T}$ independent on which ramification point is chosen as zero.

Remark 1.2 Since the permuting of the 3 sheets is an automorphism, if a (nonramification) point in $\mathrm{T}$ is of $3 \mathrm{~m}$-torsion, then the remaining two points in its fiber are $3 \mathrm{~m}$-torsion as well. So by lemma 1.1 the set of images

$$
P_{M}^{1}[3 m]=f(T[3 m]) \backslash M
$$

(where $M=\operatorname{branch}(\mathbf{f})$ is the branch locus) is a well defined set (independent of any choices and invariant under isomorphisms of marked lines) consisting of $\left((3 \mathrm{~m})^{2}-\right.$ $3) / 3=3 m^{2}-1$ distinct points. We call $\mathrm{P}_{M}^{1}[3 \mathrm{~m}]$ the set of equianharmonic $3 \mathrm{~m}$ torsion parameters relative to the markings $M$, or simply the set of equianharmonic $3 m$-torsion parameters (denoted $\left.\mathrm{P}^{1}[3 \mathrm{~m}]\right)$ if $\mathrm{M}=\{0,1, \infty\}$.

Corollary 1.3 Let $\mathrm{T}$ be an elliptic curve and $\mathbf{f}: \mathrm{T} \rightarrow \mathrm{P}^{1}$ a morphism of degree 3 with triple ramification at 3 points, and let $M=\operatorname{branch}(f)$. The set $f^{-1}(M \cup$ $\left.\mathrm{P}_{\mathrm{M}}^{1}[3 \mathrm{~m}]\right)$ is a translate of the subgroup of $3 \mathrm{~m}$-torsion.

We will be interested in the explicit determination of $\mathrm{P}^{1}[3 \mathrm{~m}]$. The first case corresponds to 3 -torsion points, $m=1$, for which there are $3 \cdot 1^{2}-1=2$ equianharmonic torsion parameters. Invariance under the action of $S_{3}$ is enough to determine $P_{M}^{1}[3]$ as the Hessian pair of the marking $M$, which can also be characterized as the two points which together with $M$ form an equianharmonic set (i.e., with cross ratio a cube root of 1). For $M=\{0,1, \infty\}$ one gets $\mathrm{P}^{1}[3]=\left\{-\boldsymbol{\varepsilon}-\varepsilon^{2}\right\}$ (this is the only set of 2 points invariant for both $t 7 \rightarrow t^{-1}$ and $\left.t 7 \rightarrow 1-t\right)$. $P^{1}[6]$, although more involved, can be computed using the $S_{3}$-action as well, but this is not the case for higher $\mathrm{m}$. Below we compute $\mathrm{P}^{1}[6]$ from the definition, a method that does generalize to all $\mathrm{m}$.

Theorem 1.4 (Halphen, $[11, \S 3]$ ) Consider each pencil $C_{t}, t \in T$ as a marked line, where the marking consists of the three singular members $C_{t_{u}}$ with $u \in\{0,1, \infty\}$. Denote $C_{t}[3]$ the set of two cubics in the pencil that correspond to equianharmonic 3-torsion parameters. Then

$$
C_{h}[3] \cup C_{v}[3] \cup C_{\delta}[3] \cup C_{\gamma}[3]
$$


is the locus of 9-torsion points of curves in the Hesse pencil.

Using the fact that $\mathrm{P}^{1}[3]=\left\{-\boldsymbol{\varepsilon}-\varepsilon^{2}\right\}$, the 8 Halphen cubics can be explicitly written as follows.

$$
\begin{aligned}
& C_{\mathrm{v}-\varepsilon}=x^{3}+\varepsilon^{2} y^{3}+\varepsilon z^{3} \quad-\varepsilon_{-\varepsilon^{2}}=x^{3}+\varepsilon y^{3}+\varepsilon^{2} z^{3} \\
& \mathrm{C}_{\mathrm{h}_{-\varepsilon}} \mathrm{C}_{\mathrm{v}} \quad{ }_{-\varepsilon^{2}}=x \mathrm{y}^{2}+\mathrm{x}^{2} \mathrm{z}+\mathrm{yz} \mathrm{z}^{2} \\
& C_{\delta_{-\varepsilon}}=x^{2} y+y^{2} z+x z^{2} \quad C_{h}^{-\varepsilon^{2}}=x y^{2}+\varepsilon^{2} x^{2} z+\varepsilon y z^{2} \\
& C_{\gamma-\varepsilon}=x^{2} y+\varepsilon^{2} y^{2} z+\varepsilon x z^{2} \quad{ }_{-\varepsilon^{2}}=x y^{2}+\varepsilon x^{2} z+\varepsilon^{2} y z^{2} \\
& =x^{2} y+\varepsilon y^{2} z+\varepsilon^{2} x z^{2} \\
& \mathrm{C}_{\gamma}
\end{aligned}
$$

This same list also arises in a somewhat different way in $[2,10]$ and $[1$, Proposition 5.2], where a modern account of Halphen's theorem is given. In addition to computing the 9-torsion of members of the Hesse pencil, the Halphen cubics are special in their Hesse singular point cubic pencils, in the following ways. First, the base points of the pencil are a translate (with respect to the group law of the Halphen cubic) of its set of 3-torsion points; and second, they intersect one another only in base points, tangentially in sets of three (from distinct pencils). It is these latter properties that we seek to generalize in higher order Halphen cubics. Denote $C_{t}[3 \mathrm{~m}]$ the union of the $3 m^{2}-1$ cubics in the pencil $C_{t}$ corresponding to equianharmonic $3 \mathrm{~m}$-torsion parameters, and call them the Halphen cubics of order $\mathrm{m}$. In order to better describe their intersections we consider the blow up $\mathrm{X} \rightarrow \mathrm{P}^{2}$ at the 12 points of $V$, and denote $\tilde{\tau_{t}}[3 \mathrm{~m}]$ the strict transforms. Denote $L$ the pullback to $X$ of the class of a line, $E_{t_{u}}$ the exceptional divisor above the point $t_{u}, E$ the sum of all 12 exceptionals,

$$
E_{t}=\underbrace{X}_{t_{u}^{0} \in \Lambda_{t}} E_{t_{u}^{0}}=\underset{\substack{t^{0}=t \\ u \in\{0,1, \infty\}}}{X} E_{t_{u}^{0}}
$$

and

the divisor above the 9 point set $\Lambda_{t}$; thus each Hesse singular point cubic pencil can be written $C_{t}=\left|3 H-E_{t}\right|$. Each exceptional divisor $E_{t_{u}}$ carries a natural marking

$$
\mathrm{M}_{\mathrm{t}_{\mathrm{u}}}=\mathrm{E}_{\mathrm{t}_{\mathrm{u}}} \cap \widetilde{\mathrm{C}}_{\mathrm{t}_{\mathrm{u}}}
$$

consisting of the directions of the three lines in the dual Hesse configuration going through the point $t_{u}$ (these are the component lines of $C_{t_{u}}$ ). In the sequel, unless explicitly specified, the equianharmonic torsion points on $\mathrm{E}_{\mathrm{t}_{\mathrm{u}}}$ will always be considered with respect to the natural marking, and we denote them $E_{t_{u}}[3 m]=\left(E_{t_{u}}\right)_{M_{t_{u}}}[3 m]$.

Note that, for each $t^{0}=t$ we have

$$
E_{t_{u}} \cap \quad \widetilde{C}_{t_{0}^{0}} \cup \widetilde{C}_{t_{1}^{0}} \cup \widetilde{C}_{t_{\infty}^{0}}=E_{t_{u}} \cap \widetilde{C}_{t_{u}}=M_{t_{u}} .
$$

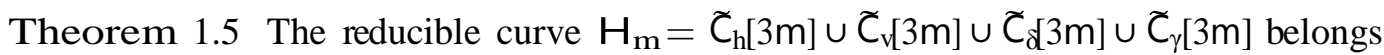
to the linear system $\left|12\left(3 m^{2}-1\right) L-9\left(3 m^{2}-1\right) E\right|$, and has the following singularities:

1. $3 m^{2}-1$ ordinary triple points on each $E_{t_{u}}$, for a total of $12\left(3 m^{2}-1\right)$ triple points;

2. $9\left(3 m^{2}-1\right)\left(m^{2}-1\right)$ ordinary quadruple points off $E$.

Moreover, each component of the curve passes through 9 of the triple points and $9\left(m^{2}-1\right)$ of the quadruple points, which together constitute a translate of its $3 m-$ torsion subgroup. 
Proof. The linear equivalence class is clear from the fact that $C_{t}[3 m] \sim\left(3 m^{2}-\right.$ 1) $\left(3 L-E_{t}\right)$.

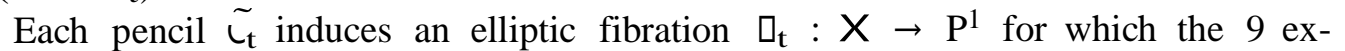
ceptional components above $\Lambda_{t}$ are sections. For every exceptional component $E_{t_{u}}$, three of the fibrations have it as a section (those $\square_{t^{0}}$ with $t^{0}=t$ ), and for the remaining fibration $\square_{t}$, it is a component of a fiber, with multiplicity 3 . Indeed,

$$
\mathrm{u}_{\mathrm{t}}^{-1}\left(\mathrm{u}_{\mathrm{t}}\left(\mathrm{C}_{\mathrm{t}_{\mathrm{u}}}\right)\right)=\widetilde{\mathrm{C}}_{\mathrm{t}_{\mathrm{u}}}+3 \mathrm{E}_{\mathrm{t}_{\mathrm{u}}}
$$

because $C_{t_{u}}$ has multiplicity 3 at $t_{u}$.

Any two pencils among the 4 share 6 base points, and hence have intersection number $3^{2}-6=3$. The restriction of $\square_{h}, \square_{\delta}$ and $\square_{\gamma}$ to any smooth curve $\widetilde{C}_{v s} s \in$ $C \backslash\{0,1\}$ in the pencil $\tilde{\sim}_{v}$ gives therefore a morphism of degree $3,\left.\square_{t}\right|_{\widetilde{C}_{v_{s}}}: \widetilde{C}_{v, s} \rightarrow E_{v_{0}}$. Since $t_{u}$ is a base point of $C_{v}$ for each $t=v$ and $u \in\{0,1, \infty\} \widetilde{C}_{v_{s}}$ meets $E_{t_{u}}$ at a point $p=p_{t_{u}}$ and by (4),

$$
\left.\mathrm{a}_{\mathrm{t}}\right|_{\widetilde{\mathrm{C}}_{\mathrm{vs}}}{ }^{-1}\left(\mathrm{C}_{\mathrm{t}_{\mathrm{u}}} \cap \mathrm{E}_{\mathrm{v}_{0}}\right)=\widetilde{\mathrm{C}}_{\mathrm{h}_{\mathrm{s}}} \cap \widetilde{\mathrm{C}}_{\mathrm{t}_{\mathrm{u}}}+3 \mathrm{E}_{\mathrm{t}_{\mathrm{u}}}>3 \mathrm{p},
$$

but since the degree of the map is 3 , the inequality must in fact be an equality (so $\widetilde{C}_{\mathrm{h}_{\mathrm{s}}}$ meets no point on $\widetilde{C}_{t_{u}}$ and the intersection with $E_{t_{u}}$ is transversal). This holds for all $u \in\{0,1, \infty\}$, so $\left.\square_{t}\right|_{\widetilde{C}_{h_{s}}}$ has triple ramification above $M_{h_{0}}$. As the action of the Hesse group on $T$ is 2-transitive, for every $t=t^{0}$ the restriction of $\mathrm{D}_{t}$ to a fiber $\widetilde{C}_{\mathbf{s}^{0}}$ has triple ramification above $M_{t_{0}^{0}}$. Since $\left.\nabla_{t}\right|_{t_{t}}$ has no additional ramification (by RiemannHurwitz) all intersections between $\widetilde{C}_{t_{s}^{0}}$ and nonsingular fibers of $\mathrm{Q}_{\mathrm{t}}$ are transversal. Therefore, intersections between components of $\mathrm{H}_{\mathrm{m}}$ (which are nonsingular fibers of the pencils) are transversal; this means that all multiple points of $\mathrm{H}_{\mathrm{m}}$, which are generated by such intersections, are ordinary. Moreover, by corollary 1.3,

$$
\widetilde{C}_{t_{s}^{0}} \cap\left(\widetilde{C}_{t}[3 m] \cup E_{t_{0}} \cup E_{t_{1}} \cup E_{t_{\infty}}\right)={D_{t}}_{t_{t_{t}}}^{-1} \quad E_{t_{0}^{0}}[3 m] \cup M_{t_{0}^{0}}
$$

consists of the $3 \mathrm{~m}$-torsion points of $\mathrm{C}_{\mathrm{t}_{\mathrm{s}}^{0}}$ up to translation, for each $\mathrm{t}^{0}=\mathrm{t}$.

By construction, for each $t=t^{0}$, the intersection $\widetilde{C}_{t}[3 m] \cap E_{t_{0}^{0}}$ consists of the equianharmonic $3 \mathrm{~m}$-torsion parameters $\mathrm{E}_{\mathrm{t}_{0}^{0}}[3 \mathrm{~m}]$, i.e., through each point of $\mathrm{E}_{\mathbf{t}_{0}}[3 \mathrm{~m}]$ there are three components of $\mathrm{H}_{\mathrm{m}}$, one in each pencil $\mathrm{C}_{\mathrm{t}}, \mathrm{t}=\mathrm{t}^{0}$. These points are therefore triple points of $\mathrm{H}_{\mathrm{m}}$, and there are $3 \mathrm{~m}^{2}-1$ such points on each of the 12 exceptional components.

Taking into account the linear equivalence class of the $\underset{\sim}{C_{t}}$ and the intersection product on $X$, we see that besides the triple points, each pair ${ }_{[3 \mathrm{~m}],}^{\sim}{ }_{0}[3 \mathrm{~m}]$ intersect at $9\left(3 m^{2}-1\right)\left(m^{2}-1\right)$ additional points. The proof will be complete by showing that these belong to the two remaining $\widetilde{\tau_{t} 0[3 \mathrm{~m}]}$ 's. We accomplish this by proving that, for every component $\widetilde{\mathrm{C}}_{\mathrm{v}_{\mathrm{S}}}$ of $\tilde{\mathrm{C}}_{\mathrm{v}}[3 \mathrm{~m}]$, the sets

$$
\begin{aligned}
& A_{h}=\tilde{C}_{v_{s}} \cap\left(\tilde{\iota}_{h}[3 m] \cup E_{h_{0}} \cup E_{h_{1}} \cup E_{h_{\infty}}\right) \\
& A_{\delta}=\widetilde{C}_{v_{s}} \cap\left(\tilde{\tau_{\delta}}[3 m] \cup E_{\delta_{0}} \cup E_{\delta_{1}} \cup E_{\delta_{\infty}}\right)
\end{aligned}
$$

are equal; then

$$
\widetilde{C}_{\mathrm{v}}[3 \mathrm{~m}] \cap{\widetilde{C_{\mathrm{h}}}}_{3}[3 \mathrm{~m}] \backslash \mathrm{E}={\widetilde{\mathrm{C}_{\mathrm{v}}}}_{\mathrm{V}}[3 \mathrm{~m}] \cap{\widetilde{\mathrm{C}_{\delta}}}_{[}[3 \mathrm{~m}] \backslash \mathrm{E}
$$

and by symmetry all pairs $\widetilde{C_{t}}[3 m], \widetilde{C_{t^{0}}}[3 \mathrm{~m}]$ intersect at the same set of $9\left(3 \mathrm{~m}^{2}-\right.$ $1)\left(m^{2}-1\right)$ points, which finishes the proof. 
Indeed, by corollary 1.3 both $A_{h}$ and $A_{\delta}$ are obtained from the set of $3 \mathrm{~m}$-torsion points of $\widetilde{\mathrm{C}}_{\mathrm{v}_{\mathrm{s}}}$ by suitable translations according to the group law in $\widetilde{\mathrm{C}}_{\mathrm{v}_{\mathrm{s}}}$, therefore $A_{h}=t_{p}\left(A_{\delta}\right)$ for some point $p \in \widetilde{C}_{v_{s}}$. Since $\widetilde{C}_{v_{s}}$ meets $E_{\gamma_{0}}$ at one of the triple points, which must also belong to $\widetilde{C}_{h}[3 \mathrm{~m}]$ and $\widetilde{C}_{\delta}[3 \mathrm{~m}]$, it follows that $A_{h} \cap A_{\delta}$ is nonempty. Therefore $p$ is of $3 m$-torsion, and $A_{h}=A_{\delta}$ as claimed.

Explicit computation of the equianharmonic torsion parameters. Our method to compute the higher order Halphen cubics geometrically is based on the following remark.

Remark 1.6 The plane cubic curve $C=x^{3}+y^{3}-z^{3}$ is a $j$-invariant 0 curve, and each of the three lines $L_{1 * * *}$ in the dual Hesse configuration going through the point $v_{1}=(0,1,0)$ is a flex line for $C$ (i.e., tangent to $C$ at a flex point). For brevity, in this section we denote these lines simply

$$
L_{1}=-\square^{2}(z-x), \quad L_{0}=\square z-x, \quad L_{w}=z-\square x
$$

(With respect to the equations $L_{1 * * *}$ above, a product with adequate constant coefficients was done so that $L_{0}+L_{\infty}=L_{1}$ ). Thus the linear series on $C$ given by the pencil of lines through $v_{1}$ defines a morphism $C \rightarrow E_{v_{1}}$ with triple ramification above the points corresponding to the directions of the $L_{u}$, which form exactly the set $\mathrm{M}_{\mathrm{v}_{1}}$; and the equianharmonic torsion parameters (with respect to $\mathrm{M}_{\mathrm{v}_{1}}$ ) can be computed as the projections to $E_{v_{1}}$ of the torsion points on $C$.

Once the $n$-torsion points on $C$ are known, their projections to $E_{v_{1}}$ (which means their $(x, z)$ coordinates) are the equianharmonic $n$-torsion parameters. The torsion points can be found in principle solving algebraic equations involving so-called division polynomials [14], so the method works uniformly for all $\mathrm{n}$. In this section we will determine the equianharmonic 6-torsion parameters, where we can find the required 6-torsion points using a more geometric method. We then produce the 44 Halphen cubics of order 2. These include the 8 order 1 cubics, so we have 36 still to find.

Lemma 1.7 The 6-torsion points of $C$ are obtained by adding (using the group law on $C$ ) each of the nine points

$$
\begin{aligned}
& (1,0,1),(1,0, \square),\left(1,0, \square^{2}\right),(1,-1,0),(1,-\square, 0),\left(1,-\square^{2}, 0\right),(0,1,1),(0,1, \square),(0,1, \\
& \left.\square^{2}\right)
\end{aligned}
$$

to each of the four points

$$
(1,0,1),(1,-b,-1),(1,-\square b,-1),\left(1,-\square^{2} b,-1\right)
$$

where $b^{3}-2=0$.

Proof. The 6-torsion points can be obtained by adding 3-torsion points and 2-torsion points. The 3-torsion points are the flex points, which as noted above, are the nine points given. As for the 2-torsion points, note first that $(1,0,1)$ is a flex. Regarding it as the identity for the group law on $C$, the 2-torsion points on $C$ are the lines tangent to $C$ which go through the identity (i.e., through $(1,0,1))$. One can check that the required points are the four points given. Using the group law on $C$ one can now find all 36 of the 6-torsion points. 
Projections from 6-torsion points. Three of the 6-torsion points are the ramification points of the projection, and 6 of them correspond to the $n=3$ case. The other 27 come from taking a line through a 2-torsion point and one of the nine 3 -torsion points. One then finds the line through each of these 27 points and the point $\mathrm{v}_{1}$. This gives 9 lines through $\mathrm{v}_{1}$ :

$$
\begin{aligned}
& x-(1 / 2) b^{2} z, \quad x-(1 / 2) \square b^{2} z, \quad x-(1 / 2) \square^{2} b^{2} \\
& z x-b z, \quad x-\square b z, \quad x-\square^{2} b z \\
& x+z, \quad x+\square z, \quad x+\square^{2} z
\end{aligned}
$$

The ramification points map to the lines $L_{i}$, and the lines above (up to product with a constant, in the same order) can be written as:

$$
\begin{aligned}
& (b \square-1) L_{0}-\square\left(b \square^{2}-1\right) L_{\omega},(b-1) L_{0}-\square\left(b \square-\quad, \quad\left(b \square^{2}-1\right) L_{0}-\square(b-1) L_{\infty}\right. \\
& \text { 1) } \mathrm{L}_{\infty} \\
& \left(b \square^{2}-1\right) L_{0}-a^{2}(b \square-1) L_{,}, \quad(b-1) L_{0}-a^{2}\left(b \square^{2}-1\right) L, \quad(b \square-1) L_{0}-a^{2}(b-1) L^{w} \\
& \mathrm{~L}_{0}-\mathrm{L}_{\infty}, \quad 2 \mathrm{~L}_{0}+\mathrm{L}_{\infty}, \quad \text { w } \quad \mathrm{L}_{0}+2 \mathrm{~L}_{\infty}
\end{aligned}
$$

Now the equianharmonic torsion parameters $\mathrm{P}^{1}[6] \backslash \mathrm{P}^{1}[3]$ can be obtained as the ratios between the coefficients of $L_{\infty}$ and $L_{0}$ in the previous list. In the same order again:

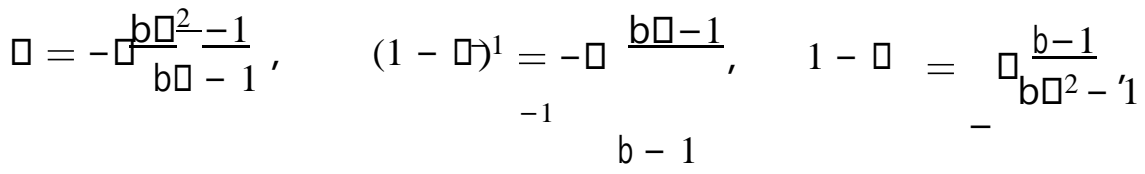

$$
\begin{aligned}
& \square^{-1}=-\square_{b}^{2} \frac{b \square-1}{\square^{2}-1}, \quad\left(1-\square^{-1}\right)^{-1}=-\square^{2} \frac{b \square-1}{b-1}, \quad 1-\square=-\square_{b \square-1}^{2} \underline{b-1}, \\
& -1 \text {, } \\
& \frac{1}{2} \\
& 2
\end{aligned}
$$

As an aside we note that 3 of them are defined over Q; they form one orbit under the action of $S_{3}$ generated by $t \rightarrow 1-t$ and $t 7 \rightarrow t^{-1}$. The remaining 6 form another orbit, which consists of the roots of the irreducible invariant polynomial $P(x)=x^{6}-3 x^{5}+5 x^{3}-3 x+1=\left(x^{2}-x-1\right)^{3}+2$, with the property that $\mathrm{Q}[\mathrm{G}]=\mathrm{Q}[\mathrm{\square}, \mathrm{b}]$ is the splitting field of $\mathrm{x}^{3}+2$ (see [7, page 59]).

The 36 new Halphen cubics. Now here are the 36 cubics we get, normalized to obtain a simple expression (i.e., the polynomial $C_{v_{\tau}}$ as given on the list is actually a scalar multiple of $\left.C_{v_{0}}+\square C_{v_{\infty}}\right)$.

$$
\begin{aligned}
& C_{v_{\tau}}=(b-a) x^{3}+a^{2}(b-1) y^{3}+(b a-1) z^{3} \\
& C_{h_{\tau}}=x^{2} y-\square^{2} b x y^{2}-\square^{2} b x^{2} z+y^{2} z+x z^{2}-a^{2} b y z \\
& C_{\delta_{\tau}}=x^{2} y-b x y^{2}-\square^{2} b x^{2} z+\square^{2} z+{ }^{2}-\square b y z \\
& C_{\gamma_{\tau}}=x^{2} y-\square^{2} b x y^{2}-b x^{2} z+{ }^{2} z+\square^{2} x z^{2}-\square b y z \\
& C_{v_{(1-\tau)-1}}=\square(b \square-1) x^{3}+\square(b-a) y^{3}+(b-1) z^{3} \\
& C_{h_{(1-\tau)-1}}=x^{2} y-\square b x y^{2}-a b x^{2} z+y^{2} z+x z^{2}-0 b y z^{2} \\
& C_{\delta_{(1-\tau)-1}}=x^{2} y-a^{2} b x y^{2}-\square b x^{2} z+\square^{2} y^{2} z+\square x z^{2}-b y z^{2} \\
& C_{\gamma_{(1-\tau)-1}}=x^{2} y-\square b x y^{2}-\square^{2} b x^{2} z+\square y^{2} z+\square^{2} x z^{2}-b y z^{2}
\end{aligned}
$$




$$
\begin{aligned}
& C_{v_{1-\tau}-1}=\square(b-1) x^{3}+\left(b-\square^{2}\right) y^{3}+\left(b \square^{2}-1\right) z^{3} \\
& C_{h_{1-\tau}-1}=x^{2} y-b x y^{2}-b x^{2} z+y^{2} z+x z^{2}-b y z^{2} \\
& C_{\delta_{1-\tau-1}}=x^{2} y-\square b x y^{2}-b x^{2} z+\square^{2} y^{2} z+\square x z^{2}-\square^{2} b y z^{2} \\
& C_{\gamma_{1-\tau}-1}=x^{2} y-b x y^{2}-\square b x^{2} z+\square y^{2} z+\square^{2} x z^{2}-\square^{2} b y z^{2} \\
& C_{v_{\tau}-1}=\left(b-a^{2}\right) x^{3}+a(b-1) y^{3}+\left(b a^{2}-1\right) z^{3} \\
& C_{h_{\tau-1}}=2 x^{2} y-a^{2} b^{2} x y^{2}-a^{2} b^{2} x^{2} z+2 y^{2} z+2 x z^{2}-a^{2} b^{2} y z^{2} \\
& C_{\delta_{\tau}-1}=2 x^{2} y-b^{2} x y^{2}-\square^{2} b^{2} x^{2} z+2 \square^{2} y^{2} z+2 \square x z^{2}-\square b^{2} y z^{2} \\
& C_{\gamma_{\tau-1}}=2 x^{2} y-\square^{2} b^{2} x y^{2}-b^{2} x^{2} z+2 \square y^{2} z+2 \square^{2} x z^{2}-\square b^{2} y z^{2} \\
& C_{\mathrm{v}_{(1-\tau-1}-1}=\square(b-\square) x^{3}+\square(b \square-1) y^{3}+(b-1) z^{3} \\
& C_{h_{(1-\tau-1)-1}}=2 x^{2} y-\square b^{2} x y^{2}-\square b^{2} x^{2} z+2 y^{2} z+2 x z^{2}-\square b^{2} y z^{2} \\
& C_{\delta_{(1-\tau-1)-1}}=2 x^{2} y-\square^{2} b^{2} x y^{2}-\square b^{2} x^{2} z+2 \square^{2} y^{2} z+2 \square x z^{2}-b^{2} y z^{2} \\
& C_{\gamma_{(1-\tau-1)-1}}=2 x^{2} y-\square b^{2} x y^{2}-\square^{2} b^{2} x^{2} z+2 \square y^{2} z+2 \square^{2} x z^{2}-b^{2} y z^{2} \\
& C_{v_{(1-\tau)-1}}=\square^{2}(b-1) x^{3}+(b-\square) y^{3}+(b \square-1) z^{3} \\
& C_{h_{(1-\tau)-1}}=2 x^{2} y-b^{2} x y^{2}-b^{2} x^{2} z+2 y^{2} z+2 x z^{2}-b^{2} y z^{2} \\
& C_{\delta_{(1-\tau)-1}}=2 x^{2} y-\square b^{2} x y^{2}-b^{2} x^{2} z+2 \square^{2} y^{2} z+2 \square x z^{2}-\square^{2} b^{2} y z^{2} \\
& C_{\gamma_{(1-\tau)-1}}=2 x^{2} y-b^{2} x y^{2}-\square b^{2} x^{2} z+2 \square y^{2} z+2 \square^{2} x z^{2}-\square^{2} b^{2} y z^{2} \\
& C_{v_{-1}}=x^{3}-2 y^{3}+z^{3} \\
& C_{h_{-1}}=x^{2} y+a^{2} x y^{2}+a^{2} x^{2} z+y^{2} z+x z^{2}+a^{2} y z^{2} \\
& C_{\delta_{-1}}=x^{2} y+x y^{2}+\square^{2} x^{2} z+\square^{2} y^{2} z+\square x z^{2}+\square y z^{2} \\
& C_{\gamma-1}=x^{2} y+\square x y^{2}+x^{2} z+\square y^{2} z+\square^{2} x z^{2}+\square y z^{2} \\
& C_{v_{\frac{1}{2}}}=x^{3}+y^{3}-2 z^{3} \\
& C_{h_{\frac{1}{2}}}=x^{2} y y+{ }^{2}+\square x^{2} z+y^{2} z+x z^{2}+\square y z^{2} \\
& C_{\delta_{\frac{1}{z}}}=x^{2} y+\square^{2} x y^{2}+\square x^{2} z+\nabla^{2} z+{ }^{2}+{ }^{2}+y z^{2} \\
& C_{\gamma_{\frac{1}{2}}}=x_{\square x} x^{2} y+2+\square^{2}+\square^{2} x^{2} z+{ }^{2} z+\square^{2} x z^{2}+y z^{2} \\
& C_{v_{2}}=2 x^{3}-y^{3}-z^{3} \\
& C_{h_{2}}=x^{2} z+x z^{2}+x^{2} y+z^{2} y+x y^{2}+z y^{2} \\
& \begin{array}{ll}
C_{\delta_{2}} & =x^{2} y+\quad{ }^{2}+x^{2} z+\square^{2} y^{2} z+ \\
\square x z & 2+\square^{2} y z^{2}
\end{array} \\
& C_{\gamma_{2}}=x^{2} y+x y^{2}+\square x^{2} z+z+\square^{2} x z^{2}+\square^{2} y z^{2} \\
& \square y^{2}
\end{aligned}
$$


As explained in the proof of Theorem 1.5, the singular points of the configuration $\mathrm{H}_{\mathrm{m}}$ are exactly the $3 \mathrm{~m}$-torsion points on each of its components, translated by one (arbitrary) base point of the pencil to which it belongs. In the case of $\mathrm{H}_{2}$, these 6-torsion points can be computed for each $C_{t_{\mathrm{s}}}$ by the same method above; we leave the details to the interested reader.

\section{The linear series of the Roulleau-Urzúa map}

Recall now the construction by Roulleau and Urzúa of their planar configuration of cubics (which we will eventually show to be equal to the Halphen cubics of order $\mathrm{m})$. Let as before $\mathrm{T}=\mathrm{C} / \mathrm{Z}[\mathrm{\square}]$, where $\mathrm{Q}=\mathrm{e}^{2 \pi \mathrm{i} / 6}$, be the equianharmonic elliptic curve, and let $p_{i}=\stackrel{i}{i}+\stackrel{i}{ }$ for 06 i 62 be the 3 fixed points of multiplication by

$\square^{2}$. Let $A$ be the abelian surface $A=T \times T$, and denote $\square: A \rightarrow A$ the induced automorphism defined by $(x, y) 7 \rightarrow\left(\square^{2} x, \square^{2} y\right)$, which has 9 fixed points, namely

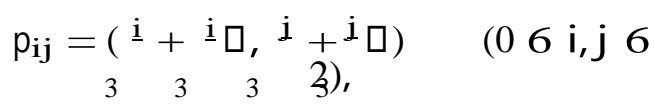

so that $p_{i j}=\left(p_{i}, p_{j}\right)$.

Divisors of particular importance on $A$ are $V=0 \times T, H=T \times 0$, the diagonal $\Delta$ and the graph $\Gamma$ of the complex multiplication by $\square$. In fact, these curves span the Néron-Severi group of $A$. Translating $V, H, \Delta, \Gamma$ by the fixed points $p_{i j}$ gives twelve curves: each of the fixed points is on four of the translates and each translate contains 3 of the fixed points, as suggested in Figure 1. Of the diagonal lines, only $\Delta$ and $\Gamma$ can be shown properly as going through three of the points $p_{\mathrm{ij}}$, but the line through points $p_{01}$ and $p_{10}$ also goes through point $p_{22}$, and in general if

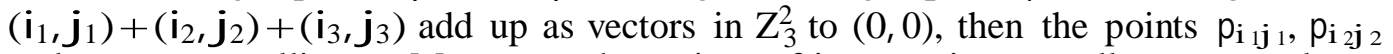
and $p_{i_{3} j_{3}}$, are collinear. Moreover, the points of intersection actually occur only at the points $p_{i j}$, contrary to how it might look in the drawing.

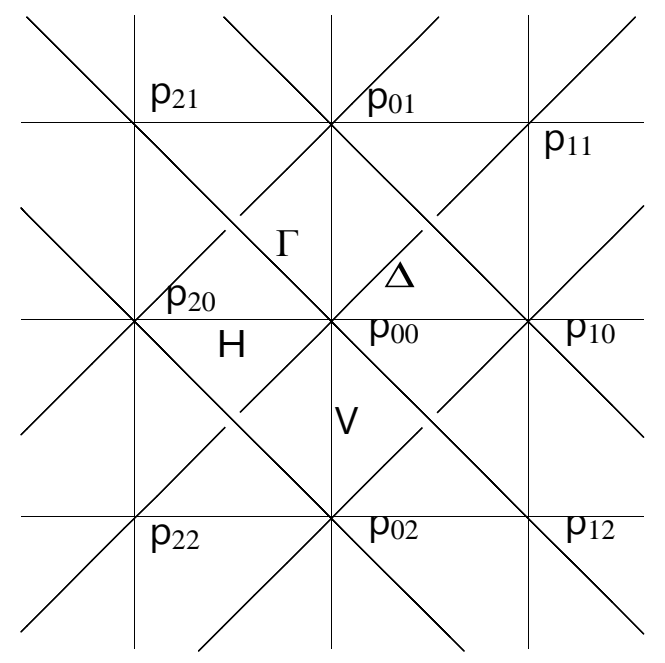

Figure 1: The curves $\mathrm{V}, \mathrm{H}, \Delta, \Gamma \subset \mathrm{A}$ and their translates by the fixed points $\mathrm{p}_{\mathbf{i j}}$

Now let $B \rightarrow A$ be the blow up at the nine points $p_{i j}$. Since these are fixed points for $\sigma$, the automorphism lifts to B. By a slight abuse of notation, we denote the lift of $\sigma$ to $B$ again by $\sigma$ (and we denote the total transforms of $V, H, \Delta, \Gamma$ to 
$B$ with the same letters). Thus we have the quotient $B \rightarrow X=B / h \square i$. Moreover, because $\square$ acts diagonally on $A, \square$ fixes tangent directions at $p_{00}$ (and hence also at each fixed point $p_{i j}$ ), so the fixed points for $\square$ acting on $B$ are exactly the points of the exceptional curves for the nine points $p_{i j}$. Thus the ramification locus for the quotient map $B \rightarrow X$ is the union of these nine exceptional curves.

Roulleau and Urzúa show in [16] that $X$ is smooth and rational, and that under the quotient $B \rightarrow X$ the images of the 12 curves obtained from $V, H, \Delta, \Gamma$ by translation are disjoint $(-1)$-curves whose contraction gives a birational morphism $\mathrm{X} \rightarrow \mathrm{P}^{2}$, representing $\mathrm{X}$ as the blow-up of $\mathrm{P}^{2}$ at the twelve points of the dual Hesse configuration. So we have a diagram

$$
\begin{array}{lll}
\mathrm{B}=\underset{\square}{\mathrm{B} l_{9}(\mathrm{~A})} & \rightarrow & \mathrm{A}=\mathrm{T} \times \mathrm{T} \\
\mathrm{X}=\mathrm{B}_{12}\left(\mathrm{P}^{2}\right) & \rightarrow & \\
\mathrm{B} & \mathrm{P}^{2}
\end{array}
$$

where the vertical map is of degree 3 and its branch locus is the union of the nine exceptional curves for the upper horizontal map, whose images are the 9 harmonic polar lines of the dual Hesse configuration.

In this section we describe the induced map $\mathrm{Q}: \mathrm{B} \rightarrow \mathrm{P}^{2}$ in terms of linear series. Then, using the action of the theta group, we determine the coordinates of the images of the 12 translates of $V, H, \Delta, \Gamma$, which will justify the choice of indices $\mathrm{v}, \mathrm{h}, \mathrm{Z}, \mathrm{Z}$ in the previous section.

\section{Theorem 2.1}

a) The morphism $\mathrm{Q}: \mathrm{B} \rightarrow \mathrm{P}^{2}$ is the map $\mathrm{C}_{\mathrm{L}}$ defined by the complete linear series |니 associated with the line bundle

$$
\mathrm{L}=\mathrm{V}+\mathrm{H}+\Delta+\Gamma-\mathrm{E}
$$

where $E$ is the sum of the nine exceptional divisors $E_{i j}$ of the blow-up $B \rightarrow A$.

b) Consider the translates $V_{i}=V+\left(p_{i}, 0\right)=V+p_{i 0}$. Then there are elliptic curves $\mathrm{N}_{01}, \mathrm{~N}_{02}, \mathrm{~N}_{12} \subset \mathrm{A}$ such that the divisors

$$
\begin{aligned}
& D_{0}:=V_{1}+V_{2}+N_{12} \\
& D_{1}:=V_{0}+V_{2}+N_{02} \\
& D_{2}:=V_{0}+V_{1}+N_{01}
\end{aligned}
$$

belong to the linear series $|\mathrm{L}|$. If we define the map $\mathrm{D}_{\mathrm{L}}: \mathrm{B} \rightarrow \mathrm{P}^{2}$ by suitably scaled sections corresponding to the divisors $D_{0}, D_{1}, D_{2}$, then the images of the 12 translates of $\mathrm{V}, \mathrm{H}, \Delta, \Gamma$ are the 12 Hesse dual points $\mathrm{v}_{\mathrm{u}}, \mathrm{h}_{\mathrm{u}}, \mathrm{D}_{\mathrm{u}}, \mathrm{a}_{\mathrm{u}}$.

The proof of Theorem 2.1 is split into several intermediate steps filling the rest of the present section. We start by showing:

Proposition 2.2 The morphism $\mathrm{\square}: \mathrm{B} \rightarrow \mathrm{P}^{2}$ is the map defined by the complete linear

series

$$
|V+H+\Delta+\Gamma-E|
$$

where $\mathrm{E}$ is as above. 
Proof. With respect to the blow up $B \rightarrow A$, the proper transforms of the curves $\mathrm{V}, \mathrm{H}, \Delta, \Gamma$ are $\mathrm{V}^{0}=\mathrm{V}-\mathrm{E}_{02}-\mathrm{E}_{00}-\mathrm{E}_{01}, \mathrm{H}^{0}=\mathrm{H}-\mathrm{E}_{20}-\mathrm{E}_{00}-\mathrm{E}_{10}, \Delta^{0}=$ $\Delta-E_{22}-E_{00}-E_{11}$ and $\Gamma^{0}=\Gamma-E_{21}-E_{00}-E_{12}$, where $E_{i j}$ is the exceptional curve for the blow up of $p_{i j}$. These curves are mutually disjoint and meet $E_{00}$ transversely. Since $V^{0}, H^{0}, \Delta^{0}$ and $\Gamma^{0}$ are preserved curvewise by $\mathrm{Q}$ and $\mathrm{E}_{00}$ is fixed pointwise, the images $\mathrm{V}^{00}, \mathrm{H}^{00}, \Delta^{00}$ and $\Gamma^{00}$ of $\mathrm{V}^{0}, \mathrm{H}^{0}, \Delta^{0}$ and $\Gamma^{0}$ under the quotient $\mathrm{B} \rightarrow \mathrm{X}$ are disjoint and meet the image $E_{00}^{0}$ of $E_{00}$ transversely. Since $V^{00}, H^{00}, \Delta^{00}$ and $\Gamma^{00}$ are exceptional curves which map to points under $X \rightarrow \mathrm{P}^{2}, \mathrm{E}_{00}^{0}$ maps to a smooth plane rational curve $C$, hence of self-intersection $C^{2}=\left(E_{00}^{0}+V^{00}+H^{00}+\Delta^{00}+\Gamma^{00}\right)^{2}=$ $\left(E_{00}^{0}\right)^{2}+4(2)+4(-1)$. But $B \rightarrow X$ is a triple cover, so $\left(\mathrm{C}^{*}\left(\mathrm{E}_{00}^{0}\right)\right)^{2}=3\left(\mathrm{E}_{00}^{0}\right)^{2}$, and so $\left(E_{00}^{0}\right)^{2}=-3$ and $C^{2}=1$, hence $C$ is a line.

$\left.{ }_{00}\right)=3 \mathrm{E}_{00}$. Thus $-9=\left(3 \mathrm{E}_{00}\right)^{2}=3\left(\mathrm{E}_{00}^{0}\right)^{2}$,

The pullback of $C$ to $B$ is $V^{0}+H^{0}+\Delta^{0}+\Gamma^{0}+3 E_{5}^{0}=V+H+\Delta+\Gamma-E$, which we denote by $L$. I.e., the map $\square$ is defined by a 3 dimensional subspace of $H^{0}(B, L)$, and the argument so far shows that $\mathrm{L}=\mathrm{V}+\mathrm{H}+\Delta+\Gamma-\mathrm{E}$. We will now prove that $h^{0}(B, L)=3$, which then implies that $\mathrm{Q}$ is defined by the complete linear series $|L|$.

First, we have $(\mathrm{V}+\mathrm{H}+\Delta+\Gamma)^{2}=12$, and therefore by Riemann-Roch on $\mathrm{A}$ we get $h^{0}(A, V+H+\Delta+\Gamma)=6$. It is therefore enough to find three fixed points $q_{1}, q_{2}, q_{3}$ of $\square$ that impose independent conditions on $|V+H+\Delta+\Gamma|$, i.e., such that there is a divisor in the linear series $|\mathrm{V}+\mathrm{H}+\Delta+\Gamma|$ passing through $\mathrm{q}_{1}$ and $q_{2}$, but not through $q_{3}$ (this suffices since the divisor $V+H+\Delta+\Gamma$ is very ample by [4, Theorem 2.3]). Consider to this end the point $x=\left(\frac{1}{3}, 0\right)$ on $A$. Lemma 2.3 implies that there is a point $z \in A$ such that the divisor $t_{x}^{*} V+H+t_{z}^{*}(\Delta+\Gamma)$ belongs to the linear series $|V+H+\Delta+\Gamma|$. Let $q_{1}$ and $q_{2}$ be any two of the three fixed points lying on $\mathrm{H}$. Clearly none of the nine fixed points lies on $t_{x}^{*} V$, and there can be at most five of them on $t_{z}^{\star}(\Delta+\Gamma)$. Therefore there exists a fixed point $q_{3}$ that lies neither on $H$ nor on $t_{z}^{*}(\Delta+\Gamma)$. The triple of points $q_{1}, q_{2}, q_{3}$ thus satisfies the required condition.

Lemma 2.3 For every pair of points $x, y \in A$ there exists a unique point $z \in A$ such that

$$
\mathrm{t}_{\mathrm{x}}^{*} \mathrm{~V}+\mathrm{t}_{\mathrm{y}}^{*} \mathrm{H}+\mathrm{t}_{\mathrm{z}}^{*}(\Delta+\Gamma) \equiv_{\operatorname{lin}} \mathrm{V}+\mathrm{H}+\Delta+\Gamma
$$

The analogous statement holds for any permutation of the curves $V, H, \Delta, \Gamma$.

Proof. Consider the homomorphism of groups

$$
\begin{aligned}
& \Phi: A \times A \times A \rightarrow P i c^{0}(A) \\
& (x, y, z) 7 \rightarrow{ }_{x}{ }^{*} V+y^{*} H+{ }_{z} t^{*}(\Delta+\Gamma)-(V+H+\Delta+\Gamma)
\end{aligned}
$$

For every pair $(x, y) \in A \times A$, the map $\Phi(x, y, \cdot)$ is a translate of the canonical homomorphism $A \rightarrow \operatorname{Pic}^{0}(A), \quad z 7 \rightarrow_{z} t^{*}(\Delta+\Gamma)-(\Delta+\Gamma)$, associated with the line bundle $\Delta+\Gamma$. Since this line bundle is of self-intersection 2 , it gives a principal polarization and therefore its canonical homomorphism is in fact an isomorphism (see [5, Prop. 2.4.9]) and thus the intersection $\operatorname{ker} \Phi \cap(\{(\mathrm{x}, \mathrm{y})\} \times$ A) consists of exactly one point.

Proposition 2.4 The map $\square$ that assigns to given points $x, y \in A$ the point $z$ as in the preceding lemma is given by

$$
\begin{aligned}
\square: A \times A & \rightarrow A \\
\left(\left(x_{1}, x_{2}\right),\left(y_{1}, y_{2}\right)\right) & 7 \rightarrow-2 x_{1}-(1+\square) y_{2},-(1+\square) x_{1}-2 y_{2}
\end{aligned}
$$


Remark 2.5 For the special case where $\mathrm{x}$ and $\mathrm{y}$ are among the nine fixed points of $\square$, we get with a calculation

$$
\square\left(p_{i j}, p_{k 1}\right)=p_{i l}
$$

In other words, we have

$$
\mathrm{t}_{\mathrm{p}_{\mathrm{ij}}}^{*} \mathrm{~V}+\mathrm{t}_{\mathrm{p}_{\mathrm{kl}}}^{*} \mathrm{H}+\mathrm{t}_{\mathrm{p}_{\mathrm{il}}}^{*}(\Delta+\Gamma) \equiv_{\text {lin }} \mathrm{V}+\mathrm{H}+\Delta+\Gamma
$$

Proof of the proposition. For a line bundle $M$ on $A$ denote as usual by $\square_{M}$ the canonical homomorphism $A \rightarrow \operatorname{Pic}^{0}(A), x 7 \rightarrow_{x} t^{*} M-M$. The point $z=\square(x, y)$ is characterized by the condition $\forall_{x}+t H_{y}+t(\Delta+\Gamma) \equiv_{\text {lin }} \quad V+H+\Delta+\Gamma$, which is

equivalent to $\square_{V}(x)+\square_{H}(y)+\square_{\Delta+\Gamma}(z)=0$. This in turn implies that

$$
\mathrm{a}(\mathrm{x}, \mathrm{y})=\mathrm{u}_{\Delta+\Gamma}^{-1}-\mathrm{\square}_{\mathrm{V}}(\mathrm{x})-\mathrm{\square}_{\mathrm{H}}(\mathrm{y})
$$

The issue therefore is to explicitly determine the canonical maps. As $\mathrm{V}+\mathrm{H}$ gives a principal polarization, $\mathrm{Q}_{\mathrm{V}+\mathrm{H}}$ is an isomorphism, and hence we can use its inverse to identify $\operatorname{Pic}^{0}(A)$ with $A$. In that sense, we will, by slight abuse of notation, denote the composed homomorphism $T \times T=A \stackrel{\varphi_{M}}{\rightarrow} \operatorname{Pic}^{0}(A) \stackrel{\varphi^{-1}+H}{\rightarrow} A=T \times T$ again by $\square_{M}$. In this setup, $\square_{V}$ and $\square_{H}$ are given by the matrices

$$
\begin{array}{lll}
1 & 0 \\
0 & 0
\end{array} \text { and } \begin{array}{ll}
0 & 0 \\
0 & 1
\end{array}
$$

respectively. We now determine the map $\square_{\Delta+\Gamma}$ in these terms. Consider to this end the isomorphism $\mathrm{g}: \mathrm{T} \times \mathrm{T} \rightarrow \mathrm{T} \times \mathrm{T},(\mathrm{x}, \mathrm{y}) 7 \rightarrow(\mathrm{x}, \mathrm{y}-\mathrm{x})$. The analytic representation of $g$ and its dual map $\hat{g}$ are

$$
\begin{array}{ccccc}
1 & 0 & \text { and } & 1 & -1 \\
-1 & 1 & 0 & 1
\end{array}
$$

We have $g^{-1}(H)=\Delta$, thus

$$
\mathrm{Q}_{\Delta}=\widehat{\mathrm{g}} \mathrm{D}_{\mathrm{H}} \mathrm{g}=\begin{array}{rr}
1 & -1 \\
-1 & 1
\end{array}
$$

We can proceed in the analogous way for $\square_{\Gamma}$ using the isomorphism $h: T \times T \rightarrow T \times T$, $(x, y) 7 \rightarrow(x, y-\square x)$. The analytic representations of $h$ and $\hat{h}$ are

$$
\begin{array}{ccccc}
1 & 0 \\
-\zeta & 1
\end{array} \text { and } \begin{array}{lll}
1 & -\zeta \\
0 & 1
\end{array}
$$

Since $\mathrm{h}^{-1}(\mathrm{H})=\Gamma$, we get

$$
\mathrm{u}_{\Gamma}=\hat{\mathrm{h}} \mathrm{D}_{\mathrm{H}} \mathrm{h}=\begin{array}{cc}
1 & -\zeta \\
-\zeta & 1
\end{array}
$$

In conclusion we find

$$
\square_{\Delta+\Gamma}=\underset{-1-\zeta}{2} 2^{-1-\bar{\zeta}}
$$

The assertion follows now from (5) using the matrices we just found.

Lemma 2.6 The divisor $\mathrm{H}+\Delta+\Gamma-\mathrm{V}$ is numerically equivalent to an elliptic curve $\mathrm{N}$. We have

$$
\square_{\mathrm{N}}=\begin{array}{cc}
1 & -1-\square^{-1} \\
-1-\square & 3
\end{array}
$$


Proof. The line bundle $H+\Delta+\Gamma-\mathrm{V}$ has self-intersection 0 and it has positive intersection with the ample bundle $\Delta+\Gamma$. This implies that its numerical class belongs to a sum of numerically equivalent elliptic curves. As its intersection with $\mathrm{H}$ is 1 , it is in fact the class of a single elliptic curve. The second assertion follows from the equation

$$
\mathrm{Q}_{\mathrm{N}}=\mathrm{Q}_{\mathrm{H}}+\mathrm{Q}_{\Delta}+\mathrm{Q}_{\Gamma}-\mathrm{Q}_{\mathrm{V}}
$$

upon using the explicit matrix representations of the maps that were worked out above.

The following statement can be useful in understanding the map $\mathrm{B} \rightarrow \mathrm{P}^{2}$, or in the construction of a basis of $H^{0}(B, L)$.

Lemma 2.7 Consider the line bundle $\mathrm{M}=\mathrm{V}+\mathrm{H}+\Delta+\Gamma$ on $\mathrm{A}$. All nine fixed points of the automorphism $\square=\left(\square^{2}, \square^{2}\right)$ are contained in the kernel $K(M)$ of $\square_{M}$ . In other words, if $D \in|M|$, then

$$
t_{x}^{*} D \in|M| \quad \text { for every } x \text { in } \operatorname{Fix}(\square)
$$

Proof. From the equation $\square_{M}=\nabla_{V}+\square_{H}+\nabla_{\Delta}+\square_{\Gamma}$ we get

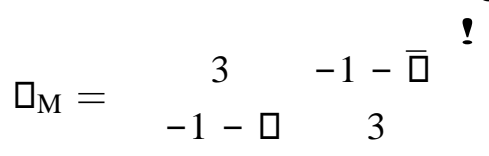

and one checks that $\square_{M} \cdot p_{i j}$ is contained in $(Z+Z \square) \times(Z+Z \square)$ for every $\mathbf{i}$ and $\mathbf{j}$.

Preimages of lines. As we know, the three translates $V_{i}=V+p_{i 0}$ map to points in $\mathrm{P}^{2}$. We would like to see the curves on $A$ which correspond to the lines ${ }_{\mathrm{ij}}$ through any two of those points. As the preimage of ${ }_{i j}$ contains $V_{i}$ and $V_{j}$, we have $\mathrm{V}+\mathrm{H}+\Delta+\Gamma=\mathrm{V}_{\mathrm{i}}+\mathrm{V}_{\mathbf{j}}+\mathrm{N}_{\mathrm{ij}}$, where the residual curve $\mathrm{N}_{\mathrm{ij}}$ is an elliptic curve (by Lemma 2.6). Its intersection numbers with the generators are

$$
\mathrm{N}_{\mathrm{ij}} \cdot \mathrm{V}=3, \mathrm{~N}_{\mathrm{ij}} \cdot \mathrm{H}=1, \mathrm{~N}_{\mathrm{ij}} \cdot \Delta=1, \mathrm{~N}_{\mathrm{ij}} \cdot \Gamma=1
$$

On the other hand, every elliptic curve on $A$ that passes through the origin arises as the image of a homomorphism $T \rightarrow A, x 7 \rightarrow(a x+b \square x, c x+d D x)$ for suitable integers $a, b, c, d$ (see [12]). Using the method from [3, Sect. 4.2] one finds that the elliptic curve $N$ corresponding to $(a, b, c, d)=(1,1,0,1)$, i.e, the image of the map $x 7 \rightarrow(x+\square x, \square x)$ has the same intersection numbers as $N_{i j}$ and is therefore numerically equivalent to $N_{i j}$. So $N_{i j}$ can be obtained from $N$ by a translation - and we determine now explicitly such a translation. The idea is this: We know that the divisor $V_{i}+V_{j}+N_{i j}$ passes through all 9 points $p_{i j}$. Since $V_{i}$ and $V_{j}$ cover 6 of them, $N_{i j}$ must pass through the remaining 3. Now, a computation shows that the intersection points of $N$ and $V_{0}$ are $p_{00}, p_{01}, p_{02}$. This implies that $\mathrm{N}=\mathrm{N}_{12}$. The other cases are obtained via translation by suitable fixed points altogether we have

$$
\begin{aligned}
& \mathrm{N}_{12}=\mathrm{N} \\
& \mathrm{N}_{02}=\mathrm{N}+\mathrm{p}_{10}=\mathrm{t}_{-\mathrm{p}_{10}}^{*} \mathrm{~N}=\mathrm{t}_{\mathrm{p}_{20}}^{*} \mathrm{~N} \\
& \mathrm{~N}_{01}=\mathrm{N}+\mathrm{p}_{20}=\mathrm{t}_{-\mathrm{p}_{20}}^{*} \mathrm{~N}=\mathrm{t}_{\mathrm{p}_{10}}^{*} \mathrm{~N}
\end{aligned}
$$


The images of the contracted translates. We know that the 12 translates of $V, H, \Delta, \Gamma$ by fixed-points of $\square$ map to points in $P^{2}$. We will use the notation

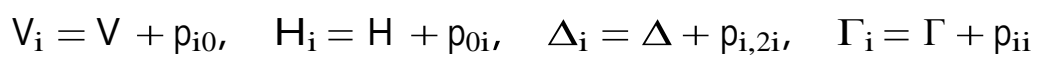

for these translates (where $06 \mathrm{i} 62$ ) and we will determine the coordinates of their image points. Consider in the linear series $\mid$ 니 the divisors

$$
\begin{aligned}
& D_{0}:=V_{1}+V_{2}+N_{12} \\
& D_{1}:=V_{0}+V_{2}+N_{02} \\
& D_{2}:=V_{0}+V_{1}+N_{01}
\end{aligned}
$$

We choose sections $s_{i} \in H^{0}(A, M)$ defining them and use these to define the map $\mathrm{D}_{\mathrm{L}}: \mathrm{B} \rightarrow \mathrm{P}^{2}$. Clearly the vertical curves $\mathrm{V}_{0}, \mathrm{~V}_{1}, \mathrm{~V}_{2}$ then map to the points

$$
\mathrm{v}_{0}=(1: 0: 0), \quad \mathrm{v}_{1}=(0: 1: 0), \quad \mathrm{v}_{\infty}=(0: 0: 1)
$$

respectively. We will now use the projective representation $K(M) \rightarrow \operatorname{PGL}\left(H^{0}(A, M)\right)$ (see [5, Chap. 6]) in order to determine the coordinates of the images of the remaining nine curves $H_{i}, \Delta_{i}, \Gamma_{i}$. By Lemma 2.7 we have Fix $\left(\square^{2}\right) \subset K(M)$, and we know that translation by fixed points leaves the condition of vanishing in these points invariant. Therefore the representation restricts to $\operatorname{Fix}\left(\mathrm{Q}^{2}\right) \rightarrow \operatorname{PGL}\left(\mathrm{H}^{0}(B, L)\right)$. In other words, each of the nine fixed points gives rise to a projective transformation of $\mathrm{P}^{2}$. Let $\mathrm{M}_{\mathrm{ij}}$ denote the projective transformation corresponding to $\mathrm{p}_{\mathrm{ij}}$. As the translation $t_{p_{10}}$ cycles the vertical translates, $V_{0} \rightarrow V_{1} \rightarrow V_{2} \rightarrow V_{0}$, we know that $M_{10}$ must be of the form

$$
M_{10}=\begin{array}{ccc}
0 & 0 & \lambda_{3} \\
\lambda_{1} & 0 & 0 \\
0 & \lambda_{2} & 0
\end{array} \text { ! }
$$

with non-zero entries $\square_{i}$. Note now that scaling the sections $s_{i}$ corresponds to a diagonal transformation on $\mathrm{P}^{2}$. We can therefore scale the $\mathrm{s}_{\mathrm{i}}$ (which leaves the coordinates in (6) invariant) in such a way that in fact $\square_{1}=\square_{2}=\square_{3}=1$, so that

$$
\mathrm{M}_{10}=\begin{array}{lll}
0 & 0 & 1 \\
1 & 0 & 0 \\
0 & 1 & 0
\end{array}
$$

The key is now the fact that the horizontal curves $\mathrm{H}_{0}, \mathrm{H}_{1}, \mathrm{H}_{2}$ are fixed under $p_{10}$. Their coordinate vectors must therefore be eigenvectors of $\mathrm{M}_{10}$. These are

$$
h_{0}=(1: 1: 1), \quad h_{1}=\left(1: \square: \square^{2}\right), \quad h_{0}=\left(1: \square^{2}: \square\right)
$$

where $\square$ denotes a primitive third root of unity. (After possibly rechoosing the origin in A they are in this order.)

Consider now the translation $t_{p_{01}}$. It fixes $V_{0}, V_{1}, V_{2}$ and therefore $M_{01}$ is of the form

$$
\mathrm{M}_{01}=\begin{array}{ccc}
\mu_{1} & 0 & 0 \\
0 & \mu_{2} & 0 \\
0 & 0 & \mu_{3}
\end{array}
$$

with non-zero entries $\mu_{i}$. Since $t_{p 01}$ maps $H_{0}$ to $H_{1}$, we have in fact

$$
M_{01}=\begin{array}{lll}
1 & 0 & 0 \\
0 & \varepsilon & 0 \\
0 & 0 & \varepsilon^{2}
\end{array}
$$


after scaling $M_{01}$ if necessary. (Here we use that the images of the $H_{i}$ are given by the coordinates, and the order, in (7).) With this information at hand, we can now also determine the coordinates of the images of the $\Delta_{i}$ and $\Gamma_{i}$. First, the diagonal translates $\Delta_{i}$ are fixed under $t_{p_{11}}$, and therefore their images are given by the eigenvectors of the matrix

$$
M_{11}=M_{10} \cdot M_{01}=\begin{array}{ccc}
0 & 0 & 1 \\
\varepsilon & 0 & 0 \\
0 & \varepsilon^{2} & 0
\end{array}
$$

Thus we get the points

$$
\square_{0}=(\square: 1: 1), \quad \square_{1}=(1: \square: 1), \quad \square_{\infty}=(1: 1: \square)
$$

(8) Here the first of these points is the image of $\Delta_{0}$, because it is this point

among

the three which is collinear with the images of $\mathrm{V}_{0}$ and $\mathrm{H}_{0}$. And finally, the graph translates $\Gamma_{\mathrm{i}}$ are fixed under $t_{p_{12}}$, which leads us to the matrix

$$
\mathrm{M}_{12}=\mathrm{M}_{01}^{2} \cdot \mathrm{M}_{10}=\begin{array}{ccc}
0 & 0 & 1 \\
\varepsilon^{2} & 0 & 0 \\
0 & \varepsilon & 0
\end{array}
$$

and the coordinates

$$
\square_{0}=\left(\square^{2}: 1: 1\right), \quad \square_{1}=\left(1: \square^{2}: 1\right), \square=\left(1: 1: \square^{2}\right)
$$

The first of these points is the image of $\Gamma_{0}$ (again by collinearity with $V_{0}$ and $H_{0}$ ). Summing up, we found that in the chosen basis of $\mathrm{H}^{0}(\mathrm{~L})$ the image points of the 12 translates are given by (6), (7), (8) and (9), and these coincide with the points in the dual Hesse configuration in standard form.

The Roulleau-Urzúa configuration. Let $n=3 m$ for some integer $m \geq 1$. Using the group of $n$-torsion points on $A$, we can translate the curves $V, H, \Delta$ and $\Gamma$ to obtain an a priori count of $4 n^{4}$ curves, 4 each at each of the $n^{4} n$-torsion points. Since each of the divisors $V, H, \Delta$ and $\Gamma$ contain $n^{2}$ of the torsion points, and thus are their own images under translation by this subgroup, there are only actually $4 n^{4} / n^{2}=4 n^{2}$ curves. The images under $\square_{L}: B \rightarrow P^{2}$ of the proper transforms under $\mathrm{B} \rightarrow \mathrm{A}$ of these curves form the Roulleau-Urzúa configuration. We can now prove that these are exactly the Halphen cubics of order $\mathrm{m}$.

Proof of Theorem 2. The n-torsion subgroup contains the order 9 subgroup consisting of the 9 points fixed with respect to the action of $\square$ on $A$. Each of the 12 curves through these 9 points (these are the curves shown in Figure 1) map to points of $\mathrm{P}^{2}$, and we found these points above. The orbits under applications of $\square$ among the remaining $4\left(n^{2}-3\right)$ curves consist of 3 curves each. Thus under $\square_{L}$ these curves map 3 to 1 to cubic curves, and the images of the $4\left(n^{2}-3\right)$ curves in $A$ are $4\left(n^{2}-3\right) / 3=4\left(3 m^{2}-1\right)$ cubic curves in $P^{2}$.

The curves in the pencils $\left(H_{u}\right),\left(\Delta_{u}\right)$ and $\left(\Gamma_{u}\right)$ meet $V_{0}, V_{1}, V_{2}$, so their images pass through the points $\mathrm{V}_{0}, \mathrm{v}_{1}, \mathrm{~V}_{\infty}$. Similarly, the curves in the pencils $\left(\mathrm{V}_{\mathrm{u}}\right),\left(\Delta_{\mathrm{u}}\right)$ and $\left(\Gamma_{\mathrm{u}}\right)$ meet $\mathrm{H}_{0}, \mathrm{H}_{1}, \mathrm{H}_{2}$, so their images pass through the points $h_{0}, h_{1}, h_{\infty}$, and so on. All together, the images of curves in $\left(\mathrm{V}_{\mathrm{u}}\right)$ pass through all 9 points in $\Lambda_{\mathrm{v}}$, so they are members of $C_{v}$, and similarly the pencils $\left(H_{u}\right),\left(\Delta_{u}\right)$ and $\left(\Gamma_{u}\right)$ map to the $\begin{array}{llllll}\text { pencils } & \mathrm{C}_{\mathrm{h}} & , & \mathrm{C}_{\delta} & \text { and } & \mathrm{C}_{\gamma}\end{array}$ 
Note that $n^{2}-3$ curves on $A$ come from each of the 4 pencils. The ones which meet $\mathrm{V}$ come from the pencils $\left(\mathrm{H}_{\mathrm{u}}\right),\left(\Delta_{\mathrm{u}}\right)$ and $\left(\Gamma_{\mathrm{u}}\right)$, with one from each pencil meeting $V$ at each of the $n^{2}-3 n$-torsion points on $V$ not fixed by $\square$. Now, $V$ maps to the point $\mathrm{v}_{0} \in \mathrm{P}^{2}$, and the triples of curves at each torsion point of $\mathrm{V}$ thus map to curves with the same tangent direction at $v_{0}=(1: 0: 0)$. The tangent directions are the $\left(n^{2}-3\right) / 3$ infinitely near images in $X$ of the $n$-torsion points on $\mathrm{V}$ not fixed by $\mathrm{Q}$. We also get 3 tangent directions corresponding to the infinitely near images of the $3 \mathrm{n}$-torsion points on $\mathrm{V}$ fixed by $\mathrm{Q}$; but these we know to be the tangent directions of the 3 lines of the dual Hesse configuration that pass through $v_{0}$, which are the images of the 3 exceptional divisors $E_{0 i}$ above the fixed points on $V$. So the restriction of the quotient map $B \rightarrow X$ to $V$ is a degree 3 morphism $\mathrm{V} \rightarrow \mathrm{E}_{\mathrm{v}_{0}}$ triply ramified above the directions of the dual Hesse lines, and therefore the tangent directions to the Roulleau-Urza cubics in $C_{h}, C_{\delta}$ and $C_{\gamma}$ are exactly the points in $E_{v_{0}}[3 m]$. So, they are indeed the Halphen cubics of order $m$ in the pencils $\mathrm{C}_{\mathrm{h}}, \mathrm{C}_{\delta}$ and $\mathrm{C}_{\gamma}$.

The same argument applied to the restriction of $\mathrm{B} \rightarrow \mathrm{X}$ to $\mathrm{H}$ proves that the Roulleau-Urzúa cubics in the remaining pencil $C_{v}$ are the Halphen cubics as well.

\section{References}

[1] Artebani, M., Dolgachev, I.: The Hesse pencil of plane cubic curves. L'Enseignement Mathématique. Revue Internationale. 2e Série 55 (2009) 235-273.

[2] Aure, A., Decker, W., Popescu, S., Hulek, K., Ranestad, K.: The geometry of bielliptic surfaces in $\mathrm{P}^{4}$. Internat. J. Math., 4 (1993) 873-902.

[3] Bauer, Th., Schulz, C.: Seshadri constants on the self-product of an elliptic curve. Journal of Algebra 320 (2008), 2981-3005.

[4] Bauer, Th., Szemberg, T.: On tensor products of ample line bundles on abelian varieties. Math. Z. 223, 79-85 (1996)

[5] Birkenhake, C., Lange, H.: Complex abelian varieties. Springer, 2004.

[6] Catanese, F., Ciliberto, C.: On the irregularity of cyclic coverings of algebraic surfaces. Geometry of complex projective varieties (Cetraro, 1990), 89-115, Sem. Conf., 9, Mediterranean, Rende, 1993.

[7] Cohen, H.: Advanced topics in computational number theory. Graduate Texts in Mathematics, 193. Springer-Verlag, New York, 2000. xvi+578 pp. ISBN: 0-387-98727-4

[8] Comessatti, A.: Sui piani tripli ciclici irregolari. Rend. Circ. Mat. Palermo 21 (1911), 369-386.

[9] Dolgachev, I. V.: Classical algebraic geometry. A modern view. Cambridge University Press, 2012.

[10] Frium, H.: The group law on elliptic curves on Hesse form, in "Finite fields with applications to coding theory, cryptography and related areas (Oaxaca, 2001), 123-151, Springer, Berlin, 2002.

[11] Halphen, G.: Recherches sur les courbes planes du troisieme degré. Math. Ann. 15, 359-379 (1879)

[12] Hayashida, T., Nishi, M.: Existence of curves of genus two on a product of two elliptic curves. J. Math. Soc. Japan 17, No. 1, 1-16 (1965)

[13] Hirzebruch, F.: Chern numbers of algebraic surfaces: an example. Math. Ann. 266, no. 3, 351-356 (1984)

[14] Lang, S.: Elliptic curves: Diophantine Analysis, Springer-Verlag, 1978.

[15] Roulleau, X.: Bounded negativity, Miyaoka-Sakai inequality and elliptic curve configurations. Preprint 2014, arXiv:1411.6996.

[16] Roulleau, X., Urzúa, G.: Chern slopes of simply connected complex surfaces of general type. Ann. Math. 182, 287-306 (2015)

[17] Silverman, J. H., The arithmetic of elliptic curves. Graduate Texts in Mathematics, 106. Springer-Verlag, New York, 1986. xii+400 pp. ISBN: 0-387-96203-4 
Thomas Bauer, Fachbereich Mathematik und Informatik, Philipps-Universität Marburg, Hans-Meerwein-Straße, D-35032 Marburg, Germany

E-mail address: tbauer@mathematik. uni-marburg. de

Brian Harbourne, Department of Mathematics, University of Nebraska, Lincoln, NE 68588-0130 USA

E-mail address: bharbourne1@un1.edu

Joaquim Roé, Departament de Matemàtiques, Universitat Autònoma de Barcelona, 08193 Bellaterra (Barcelona), Spain

E-mail address: jroe@mat. uab.cat

Tomasz Szemberg, Department of Mathematics, Pedagogical University of Cracow, Podchorążych 2, PL-30-084 Kraków, Poland

Current Address: Polish Academy of Sciences, Institute of Mathematics, Śniadeckich 8, PL-00-656 Warszawa, Poland

E-mail address: tomasz. szemberg@gmail.com 